\title{
QCD Pomeron at Linear Colliders ${ }^{1}$
}

\author{
Cong-Feng Qiao \\ II Institut für Theoretische Physik, Universität Hamburg \\ Luruper Chaussee 149, D-22761 Hamburg, Germany.
}

\begin{abstract}
Recent developments in theory on the calculation of $\gamma^{*} \gamma^{*}$ reaction at high energies, in the aim of detecting the BFKL Pomeron signals, are briefly introduced. The importance of the NLO QCD corrections to the Photon Impact Factor in the game is highlighted.
\end{abstract}

\section{INTRODUCTION}

The experimental test of the BFKL Pomeron [1] is generally considered to be an important task in strong interaction( $\mathrm{QCD}$ ) physics. In past years, it has been studied in the small-x region at HERA intensively in many respects [2]. However, because of the non-perturbative effects on both proton and final state hadronization, there still have no definite conclusions been made.

Following the pioneering application of the BFKL formalism to high energy $\gamma^{*} \gamma^{*}$ interactions at Linear Colliders by Bartels et al. [3] and Brodsky et al. [4], recently much interest has been given to this kind of reaction [5]. This process describes the scattering of two small-size projectiles, and its high energy(not too much high) behavior is expected to be described by the BFKL Pomeron. Moreover, in $\gamma^{*} \gamma^{*}$ scattering process the detection of the BFKL signal has several advantages comparing to that in the deep inelastic scattering or hadron-hadron interactions. One of them is that at Linear Colliders one can carefully calibrates the scattering angles and energies of the outgoing leptons in order to choose both interacting photons have the same or similar virtualities, and then may get rid of or suppress the effect coming from the DGLAP evolution.

\section{THE $\gamma^{*} \gamma^{*}$ SCATTERING IN BFKL FORMALISM}

Before the advent of the field theory for strong interaction, the QCD, it is well known that the Regge theory performed excellently in describing the scattering of particles at high energies with strong interaction. With getting more and more confidence in QCD being as the correct field theory for strong interaction, people began to investigate the QCD in various aspects in details, and try to compare its predictions with what

1) work supported by the Graduiertenkolleg "Theoretische Elementarteilchenphysik". 
obtained in Regge theory as well. The BFKL Pomeron, developed more than twenty years ago, is one of the counterparts of those in Regge theory,

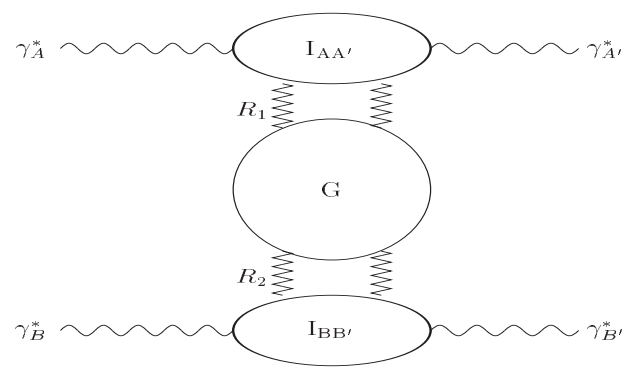

FIGURE 1. Schematic representation of the process $\gamma_{A}^{*} \gamma_{B}^{*} \rightarrow \gamma_{A^{\prime}}^{*} \gamma_{B^{\prime}}^{*}$.

In Regge limit, to Leading $\operatorname{Order}(\mathrm{LO})$ in $\log (\hat{s})$, the interaction of $e^{+} e^{-} \rightarrow e^{+} e^{-}+X$ process can be formatted as a gluon ladder with the idea of Reggeization, where the $\sqrt{\hat{s}}$ is the center-of-mass energy of $\gamma^{*} \gamma^{*}$ system. In this case the virtualities of the two photons would be large enough for the pQCD to be used reliably, but not too large to ensure it is still in the small-x domain, that is $Q_{1}^{2}, Q_{2}^{2} \gg \Lambda_{\mathrm{QCD}}^{2}$ and $x_{1}=\frac{Q_{1}^{2}}{2 q_{1} \cdot k_{2}}$, $x_{2}=\frac{Q_{2}^{2}}{2 q_{2} \cdot k_{1}} \ll 1$. For instance, the process of $\gamma_{A}^{*} \gamma_{B}^{*} \rightarrow \gamma_{A^{\prime}}^{*} \gamma_{B^{\prime}}^{*}$ can be schematically shown as Figure 1, where the upper and lower blobs represent the Pomeron Photon interactions, the Photon impact Factor(FIP) in configuration space, and to LO can be well understood in the language of Photon wave function [6], while the blob $G$ in the middle represents the Green's function of the two interacting Reggeized gluons.

\section{COMPARISON WITH THE EXPERIMENTAL DATA}
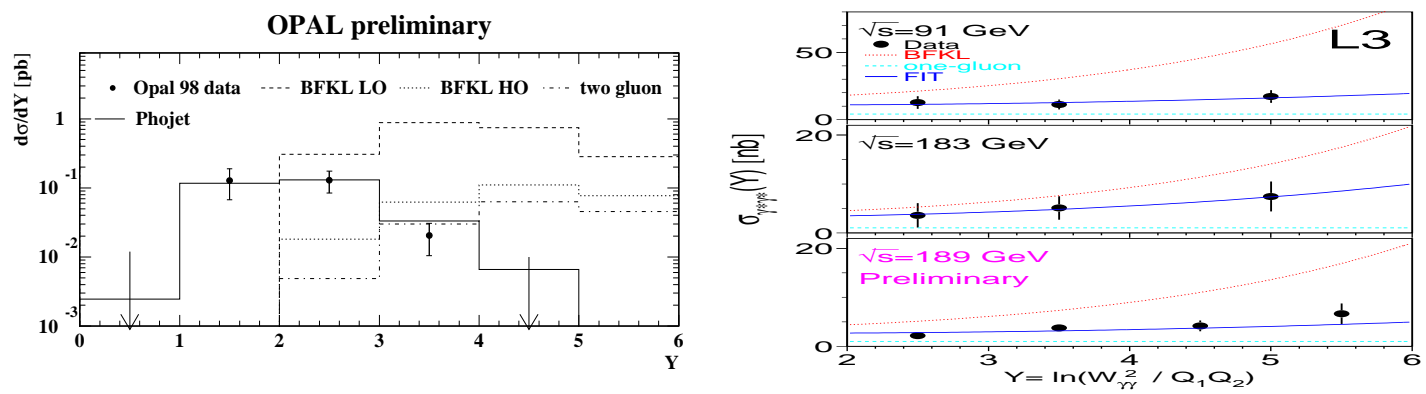

FIGURE 2. Cross-section of the the process $e^{+} e^{-} \rightarrow e^{+} e^{-} \gamma^{*} \gamma^{*}->e^{+} e^{-}+X$ as function of $Y \approx \log \frac{\hat{s}}{\mathrm{~s}_{0}}$.

So far the LO calculations have been compared to the LEP data (both OPAL and L3) [7]. Although the next-to-leading order (NLO) corrections to the BFKL equation have been obtained two year ago [8], a complete comparison of the NLO BFKL calculations with the measurements and making predictions for Future Linear Colliders (FLC) is impossible since PIF is still unknown to the same order of accuracy.

In the LO comparisons, the data from both OPAL and L3 groups lie above the two gluon exchange curve, the Born approximation, but below the BFKL prediction, as shown in Figure $2[9]$, where the $W_{\gamma \gamma}^{2}=\hat{s}$ and $\sqrt{Q_{1}^{2} Q_{2}^{2}}=s_{0}$. Because we know the NLO 
corrections to BFKL equation is very large, the comparison to go beyond the LO is necessary. By taking the BLM scale setting scheme [10], Brodsky et al. first attempted to use the NLO BFKL equation to make the prediction on $\gamma^{*} \gamma^{*}$ scattering [11], and their result is encouraging, fits to date pretty well. However, the question still remains that the NLO PIF is unknown, which for sure would cast some doubts on the result.

The reason why radiative corrections to the PIF are important is not only because the corrections may large, but also for the consistency reason of the BFKL formalism. One of the major uncertainties in doing theoretical calculations at LO is the energy scale $s_{0}$, which is not determined at LO and hence the choice of it with some arbitrariness, while its influence to the LO predictions is considerably large [3]. However, the problem in $s_{0}$ is much less serious in the full NLO calculation, or even can be fixed. Another source of uncertainty in the LO calculation is from the renormalization scale $\mu^{2}$, which also incurs large errors in the calculation [4]. As well, the scale dependence will be reduced at the NLO level.

\section{THE CALCULATION OF QCD CORRECTIONS TO PHOTON IMPACT FACTOR}

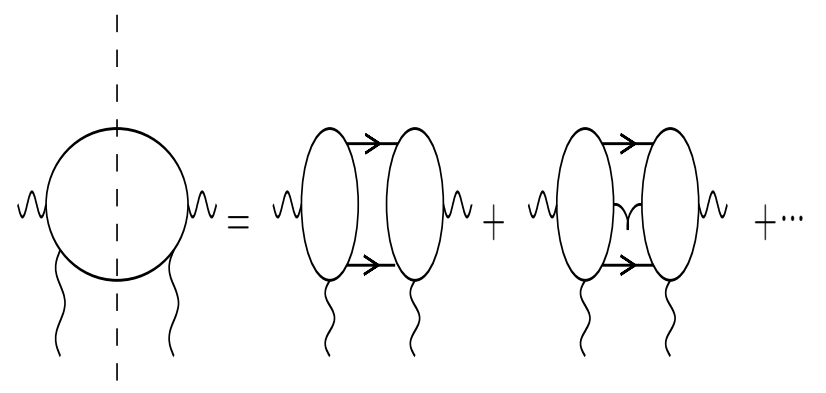

(L)

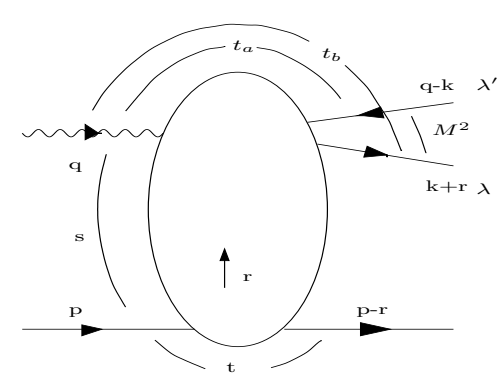

$(\mathrm{R})$

FIGURE 3. (L)Contributions to the photon impact factor; (R)Kinematics of the process $\gamma^{*}+q \rightarrow q \bar{q}+q$.

The calculations of the NLO corrections to the PIF are still on the way [12] [13]. As shown in Figure 3(L), the PIF in forward case can be obtained from the energy discontinuity of the amplitude $\gamma^{*}+$ Reggeon $\rightarrow \gamma^{*}+$ Reggeon. At LO in $\alpha_{s}$ it is simply the square of the scattering amplitude $\gamma^{*}$ and a Reggeized gluon. In NLO calculation, many possibilities of corrections should be considered. The task of calculating the NLO corrections to the PIF can be organized into three steps, (i) the calculation of the NLO corrections to the $\gamma^{*}+$ Reggeon $\rightarrow q \bar{q}$ vertex, (ii) the vertex $\gamma^{*} \rightarrow q \bar{q} g$ in LO, and (iii) the integration over the phase space of the intermediate states.

Up to now, the first of those three steps has been finished [12]. To get the NLO Reggeon-Photon Coupling, for simplicity the process $\gamma^{*}+q \rightarrow q \bar{q}+q$ in Regge limit, that is $t, Q^{2}, t_{a}, t_{b}, M^{2} \ll \hat{s}$, has been considered, as shown in Figure $3(\mathrm{R})$. The results obtained possess natures as expected, like $t_{a} t_{b}$ symmetry, etc. Because of complexity of the results in NLO Reggeon-Photon vertex, the following two steps left in calculating the NLO PIF are obviously not an easy task. 


\section{SUMMARY AND CONCLUSIONS}

We have briefly reviewed the theoretical status in the research of finding QCD Pomeron signals in $\gamma^{*} \gamma^{*}$ reaction. The current comparisons of theoretical calculations with the LEP data are encouraging, but still pre-mature. A measurement of the reaction $e^{+} e^{-} \rightarrow e^{+} e^{-}+X$ by tagging the outgoing leptons at FLC may provide an excellent test of the very important QCD prediction, the BFKL Pomeron.

Again, we would like to point out that the LEP date for the total $\gamma^{*} \gamma^{*}$ cross section clearly indicate that the LO BFKL and two-gluon model are not sufficient to describe the data. To make a consistent NLO prediction for FLC or comparison with the LEP data, the still unknown PIF with NLO QCD corrections is an inevitably ingredient.

\section{ACKNOWLEDGMENTS}

I would like to express his gratitude to J. Bartels and S. Gieseke for reading the manuscript and giving suggestions.

\section{REFERENCES}

1. E.A. Kuraev, L.N. Lipatov, V.S. Fadin, Sov. Phys. JETP 45 (1977) 199; Ya.Ya. Balitskii and L.N. Lipatov, Sov. J. Nucl. Phys. 28 (1978) 822.

2. e.g.: H. Navelet, R. Peschanski, C. Royon, S. Wallow, Phys. Lett. B385 (1996) 357; J. Bartels, V. Del Duca, A. De Roeck, D. Graudenz, M. Wusthoff, Phys. Lett. B384 (1996) 300; C. Royon, L. Schoeffel, J. Bartels, H. Jung, R. Peschanski, Hep-ph/0010015, etc.

3. J. Bartels, A. De Roeck, H. Lotter, Phys. Lett. B389 (1996) 742.

4. S.J. Brodsky, F. Hautmann, D.E. Soper, Phys. Rev. Lett. 78 (1997) 803, erratum ibid 79 (1997) 3522; ibid. Phys. Rev. D56 (1997) 6957.

5. see: J. Kwieci nski and L. Motyka, Hep-ph/0010029, and references therein.

6. A.H. Mueller, Nucl. Phys. B335 (1990) 115; N. Nikolaev, B.G. Zakharov, Z. Phys. C49 (1991) 607, C53 (1992) 331; S.J. Brodsky, P. Hoyer, and L. Magnea, Phys. Rev. D55 (1997) 5585; F. Hautmann, Z. Kunszt, D.E. Soper, Phys. Rev. Lett. 81 (1998) 3333; D. Yu. Ivanov, M. Wüsthoff, Eur. Phys. J. C8 (1999) 107; S. Gieseke and C.F. Qiao, Phys. Rev. D61 (2000) 074028.

7. J. Bartels, C. Ewerz, and R. Staritzbichler, hep-ph/0004029; A. Donnachie, S. SöldnerRembold, hep-ph/0001035.

8. V.S. Fadin, L.N. Lipatov, Phys. Lett. B 429 (1998) 127; M. Ciafaloni, G. Camici, Phys. Lett. B 430 (1998) 349; and references therein.

9. A. Donnachie and S. Öldner-Rembold, Hep-ph/0001035, and references therein.

10. S.J. Brodsky, G.P. Lepage and P.B. Machenzie, Phys. Rev. D28 (1983) 288.

11. S.J. Brodsky, V.S. Fadin, V.T. Kim, L.N. Lipatov, and G.B. Pivovarov, JETP Lett. 70 (1999) 155.

12. J. Bartels, S. Gieseke, and C.-F. Qiao, Hep-ph/0009102, to appear in Phys. Rev. D.

13. V. Fadin, D. Ivanov, and M. Kotsky, hep-ph/0007119. 